\title{
A CLINICAL METHOD OF STUDYING THE ELASTICITY OF LARGE ARTERIES
}

\author{
BY \\ JAMES CONWAY AND K. SHIRLEY SMITH \\ From the Departments of Physiology and Cardiology, Charing Cross Hospital and Medical School
}

Received March 13, 1956

Studies of the physical characteristics of large arteries (Roy, 1880; Bramwell, 1924; Hallock and Benson 1937) have shown that the aorta is more elastic in the young than in the elderly. As age advances the great vessels dilate while their distensibility diminishes and the range of pressure over which the wall is approximately elastic is reduced. These changes are the result of the tension in the arterial wall being thrown on to the collagen fibres as the elastic tissue deteriorates. Clinical studies of arterial elasticity have been made by Bramwell and Hill (1922). Their method was based upon the velocity of transmission of the pulse wave, which is determined by the physical properties of the vessel wall. The pulse wave velocity was found to increase with age (Bramwell et. al., $1923 \mathrm{~b}$ ) and with the elevation of pressure across the wall of the artery (Bramwell et. al., $1923 a$ and $c$ ). This work contributed much to the understanding of the effect of age upon the arterial tree; it confirmed, in vivo, the arterial changes, described earlier, in excised strips of aorta, and showed that loss of elasticity was not confined to the aorta and its immediate branches, but spread to the smaller and more muscular vessels, for example, from the brachial to the radial arteries. The consequence of this is seen in the well-known increase of pulse pressure with age (Master et al., 1943; Hamilton et al., 1954; Miall and Oldham, 1955). Unfortunately, the elasticity of the large arteries cannot be assessed by pulse pressure measurements alone since the latter are affected by other variables such as stroke volume, the force and duration of ventricular ejection, the peripheral resistance, and the volume of the great vessels.

The test to be described attempts to establish clinically whether the vessels forming the main arterial reservoir are mainly elastic or whether the tension is being thrown on to the inelastic collagen. The differentiation depends upon changes in pulse pressure occurring in vessels when the level of diastolic pressure is reduced with little change in stroke volume. From the data obtained by Roy (1880), Hallock and Benson (1937), and Remington et al. (1948) it is possible to predict that in young, more or less elastic arteries little change in pulse pressure would occur as the blood pressure was reduced without changing stroke volume, whereas in elderly, inelastic arteries progressive diminution in pulse pressure would be seen as the diastolic pressure fell. This theoretical basis of the test will be considered in greater detail later.

The method requires that the diastolic pressure should be reduced without significantly affecting the volume of blood ejected by the heart at each beat. Observation of the effect of nitroglycerine on the blood pressure in animal experiments suggested that this might be achieved for a limited number of heart beats, immediately after the inhalation of amyl nitrite, when an appreciable interval occurs during which the diastolic pressure falls but the heart rate is unchanged (Conway, 1955). If it were assumed that the stroke volume was not significantly changed at this early stage, then pulse pressure measurements could be made from beat to beat against a falling level of diastolic pressure and this would indicate whether the arteries were elastic or inelastic. The assumptions involved in the test will be considered later, but it so happened that tracings of the effect of amyl 
nitrite in young normal subjects and patients with hypertension, taken for another purpose, were available for study and a survey of these was sufficiently encouraging to justify pursuing the matter further. Additional clinical material was therefore obtained to investigate the reliability of the test.

\section{Material AND Method}

Fifteen normal volunteers, medical students, and thirty-seven patients, admitted to hospital with hypertension, have been studied. Patients with clinical evidence of heart failure were excluded. The test was conducted with the subjects lying comfortably on a couch while the blood pressure was measured by a condenser manometer connected by a short length of polythene tubing (approx. $20 \mathrm{~cm}$.) to a needle (SWG 25) lodged in the brachial artery, after infiltration of the skin and superficial fascia with procaine or lignocaine. The region of anæsthesia was not extended to include the artery. At frequent intervals the needle in the brachial artery was flushed through with heparinized saline to ensure accurate transmission of the pulse pressure. The condenser manometer was also checked repeatedly against known static pressures. The natural frequency of the system was approximately 20 cycles per second. The electrocardiogram and the respiration were also recorded in many instances. Records were made with ink-writing oscillographs or photographically with mirror galvomanometers. Care was taken to compare the pulse pressures in equivalent heart beats before and at the onset of the depressor effect of amyl nitrite, particular attention being given to the duration of the cardiac cycle and the phase of respiration.

After recording the initial blood pressure tracing, a crushed phial or a container of amyl nitrite was held under the subject's nose for one or two breaths and then withdrawn. The subject was clearly instructed not to " sniff" the vapour, since this would affect the venous return to the heart. In 22 subjects ( 9 normal and 14 with hypertension) the test was repeated more than once, and in 4 subjects an opportunity was available to repeat the test on another occasion. It was also possible to repeat the test after the administration of hexamethonium (20-30 mg. intravenously) in 7 normal subjects and 11 patients with hypertension.

\section{RESULTS}

The response to the inhalation of amyl nitrite, like that to an injection of nitroglycerine, may be divided into three physiological phases. The blood pressure tracing in the first phase shows a falling diastolic pressure for a limited number of heart beats without change in heart rate. The effect of amyl nitrite is seen therefore without the interference of the regulatory reflexes from the aorta and carotid sinus. After a certain fall in blood pressure the reflexes come into play and the second phase begins with tachycardia and vaso-constriction which limits the fall in pressure and rapidly restores it to normal. The third phase then follows during which the pressure is maintained at its initial level by the regulatory reflexes in spite of the continued depressor effect of the drug (Conway, 1955).

Amyl nitrite was given by inhalation in preference to intravenous injections in order to minimize the effect upon the pulmonary circulation and hence upon the filling of the left ventricle. The inhalation itself unfortunately had its own disadvantages, since it sometimes raised the blood pressure and affected the heart rate in some nervous patients. This made the tracings difficult to analyse since pulse pressure measurements preceding the inhalation could not be used for comparison with those occurring with the falling blood pressure. In these instances it was nevertheless possible to measure the pulse pressure against a declining level of diastolic pressure for an adequate number of beats in which the heart rate was steady.

The first phase of the effect of amyl nitrite has been utilized for the test of arterial elasticity since the effect of a falling diastolic pressure on the pulse pressure was seen without change in heart rate, and it was assumed that little change in stroke volume occurred at this stage. The onset of reflex tachycardia was determined very accurately even in the presence of sinus arrhythmia because the change was progressive, reaching a maximum at the time of, or just after, maximal 
depression of blood pressure. One of the main difficulties of the test was that the first phase was sometimes very short and the reduction of diastolic blood pressure small. In the normal and hypertensive subjects 84 tests were performed without the use of hexamethonium, and in these tests eight heart beats was the average number available for study, while the average reduction in diastolic pressure was $15 \mathrm{~mm}$. Hg. Fig. 1 shows the tracing obtained in a test in a normal subject

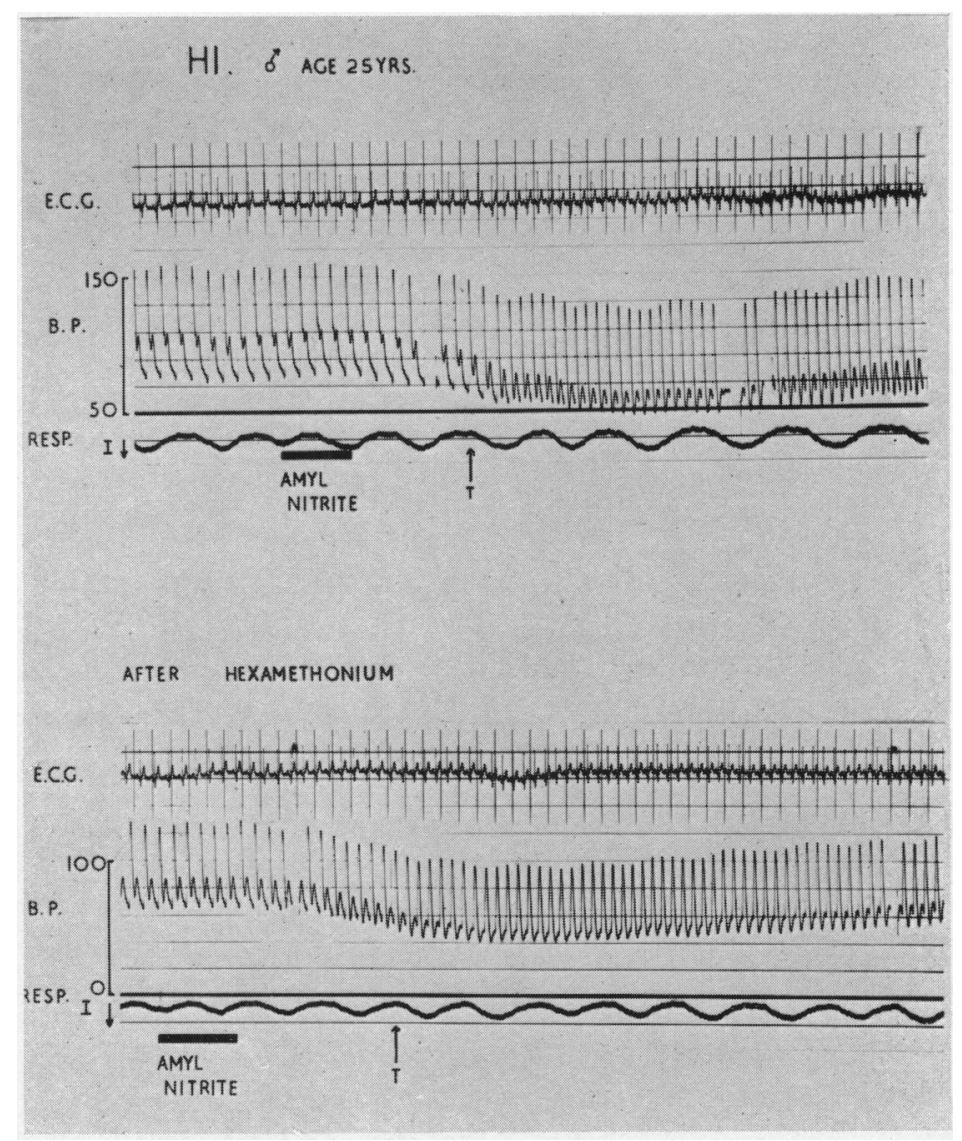

FIG. 1.-The effect of the inhalation of amyl nitrite on the blood pressure in a normal subject, before and after hexamethonium. Note gaps in the pressure tracing occur when the needle is being washed through with heparinized saline. A time-tracing is run with the electrocardiogram at $1 / \mathrm{sec}$. $\mathrm{T}$ indicates the point at which tachycardia appeared.

in which the number of beats was limited to five and it is shown that this difficulty could be overcome by partially blocking ganglionic transmission by an injection of hexamethonium dibromide (20 mg. I.V.). This diminished the vagal restraint upon the heart and delayed the onset of reflex response to the falling blood pressure. In 17 subjects in whom the test was repeated after hexamethonium, the average number of heart bests available for study was increased to 12 , with a greater average fall of the diastolic pressure to $18 \mathrm{~mm}$. Hg. Since the efficiency of the regulatory reflexes was impaired by hexamethonium it seemed wise to reduce the concentration of amyl nitrite or the duration of the inhalation. The increase in the depressor response was therefore not as great as it might have been. The combination of the two drugs produced no ill-effects. Hexamethonium was particularly useful in another way since it practically abolished changes in blood 
pressure and heart rate produced by the act of inhaling the drug and it also abolished sinus arrhythmia.

To assess changes in elasticity of the large arteries the change in pulse pressure after amyl nitrite was compared, for each heart beat, with the reduction in diastolic pressure, and the relation-

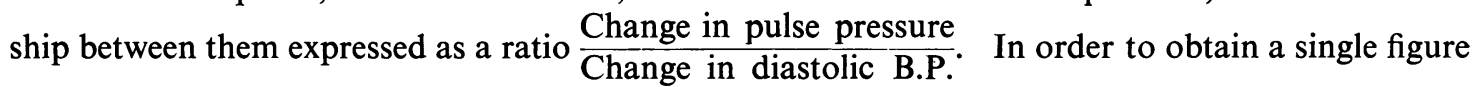
for each test, the average was taken of this ratio in the three consecutive beats in which the reduction of diastolic pressure was greatest and before tachycardia occurred; the change in pulse pressure was then expressed as a percentage of the change in diastolic pressure.

Studies of normal subjects have been confined, for the present, to the young, since it would be unlikely that they would show evidence of deterioration in elastic tissue. In age, the 15 normal subjects ranged from 17 to 32 years and in the 28 tests performed without hexamethonium, the reduction in pulse pressure with a falling diastolic pressure was very small, the average being 16 per cent of the reduction in diastolic pressure. On repetition of the test after hexamethonium in 7 subjects, the change in pulse pressure was again quite small-18 per cent. of the reduction in diastolic pressure.

All the patients studied had been admitted to hospital with hypertension but, in a few, the blood pressure at the time of the test had fallen to normal. Since the present study is concerned primarily with the presentation of the method, the findings in these patients have not been grouped separately. The 37 patients, on whom 56 tests were performed without hexamethonium showed two types of response (Fig. 2) as below.

Group I (15 patients). These gave results similar to the normals; the reduction in diastolic pressure had little effect on pulse pressure, the average reduction being 31 per cent of the change in diastolic pressure in this group. In 5 patients in whom the test was repeated after hexamethonium, the change in the pulse pressure remained at 25 per cent of the fall in diastolic pressure. This group has been called the "elastic hypertension" and the limits in this group were determined by the findings in tests on young normal subjects.

Group II (22 patients). In these, the response was entirely different. The reduction in diastolic pressure was accompanied by a conspicuous drop in pulse pressure. It was reduced by an average of 97 per cent of the change in diastolic pressure. In the 6 patients of this group in whom the test was performed after hexamethonium, the pattern of the response was unchanged, with a reduction of pulse pressure averaging 110 per cent of the fall in diastolic pressure. We have called this group the "inelastic hypertension".

When those with hypertension have been grouped as above according to their responses in the test it can be seen that patients in the inelastic Group II are on average 11 years older, and have a higher pulse pressure than those in the elastic Group I, showing that in general the separation achieved by the test agrees with clinical findings (Table). The average diastolic pressure in the inelastic group was little raised; this was because 7 out of 22 patients in this group had arteriosclerotic hypertension, with high systolic pressure but approximately normal diastolic pressure. There were no patients with arteriosclerotic hypertension among those in the "elastic" group. A clinical study of patients with hypertension will be given when further data have been accumulated, but it is of interest to note here that there were three patients in the inelastic group who were under 50 years of age: two had arterial disease, one angina and the other arteriosclerosis obliterans with gangrene of the right great toe, and the third had a history of renal tuberculosis, diagnosed 14 years earlier.

An estimate of the reliability of the test was obtained by making repeated observations on 23 subjects, 9 controls and 14 with hypertension. In four of the patients, these repetitions were performed on a separate occasion. The relationship between pulse pressure and diastolic pressure was little changed. The first test in all these subjects showed that the average fall of pulse pressure was 64 per cent of the reduction in diastolic pressure while for the subsequent tests on the same subjects the corresponding figure was 74 per cent. 

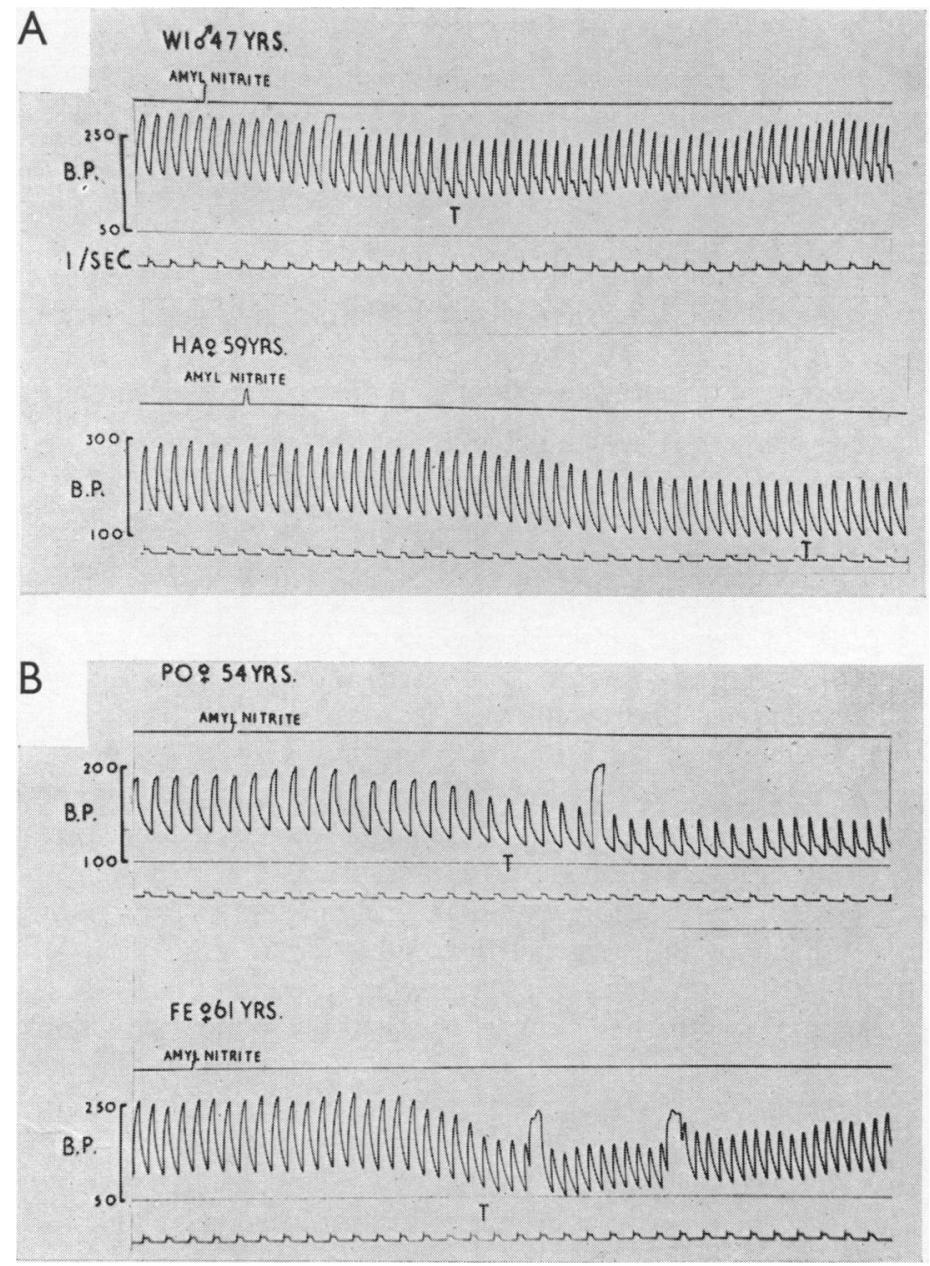

FIG. 2.-The effect of the inhalation of amyl nitrite on the blood pressure in four patients with hypertension. (A) From the group with "elastic hypertension." (B) From those with "inelastic hypertension"

Clinical Data of Normal and Hypertensive Subjects, and the effect of Amyl Nitrite on the Pulse PRESSURe

\begin{tabular}{|c|c|c|c|c|c|c|}
\hline \multirow{2}{*}{ Subjects } & \multirow{2}{*}{$\begin{array}{c}\text { Average } \\
\text { age } \\
\text { (years) }\end{array}$} & \multicolumn{3}{|c|}{ Average initial blood pressure } & \multirow{2}{*}{$\begin{array}{c}\text { Average } \\
\text { rate } \\
\text { (beats/ } \\
\text { min.) }\end{array}$} & \multirow{2}{*}{$\begin{array}{l}\text { Average change in } \\
\text { pulse pressure (as } \\
\text { percentage of the } \\
\text { reduction in } \\
\text { diastolic pressure) }\end{array}$} \\
\hline & & $\begin{array}{l}\text { Sys. } \\
\text { mm. Hg }\end{array}$ & $\begin{array}{l}\text { Dias. } \\
\mathrm{mm} . \mathrm{Hg}\end{array}$ & $\begin{array}{l}\text { Pulse P. } \\
\text { mm. Hg }\end{array}$ & & \\
\hline Normal (15) & 24 & 124 & 71 & 53 & 78 & $16 \%$ (S.D. $18 \cdot 8$ ) \\
\hline Hypertensive " elastic" (15) & $\begin{array}{c}46 \\
\text { (S.D. 9.6) }\end{array}$ & 219 & 131 & $\begin{array}{c}88 \\
\text { (S.D. 23) }\end{array}$ & 87 & $31 \%$ (S.D. 19.7) \\
\hline Hypertensive " inelastic" (22) & $\begin{array}{l}57 \\
\text { (S.D. 7.0) }\end{array}$ & 216 & 105 & $\begin{array}{c}112 \\
\text { (S.D. 18.6) }\end{array}$ & 82 & 97\% (S.D. 24.7) \\
\hline
\end{tabular}

Note. Between the two groups of hypertensive patients significant differences exist in age $(t=5 \cdot 2, P<0 \cdot 001)$, initial pulse pressure $(t=3.4, \mathrm{P}<0.01)$ and in the change in pulse pressure after amyl nitrite $(t=8.7, \mathrm{P}<0.001)$. 


\section{Discussion}

The feasibility of this new method of determining the state of the elastic tissue of the great arteries depends upon the possibility of reducing diastolic pressure without significantly affecting stroke volume. Before changes in pulse pressure after amyl nitrite can be taken to indicate changes in the elasticity of the larger arteries, certain assumptions have to be made, the most important of which are as follows.

(1) There should not be a significant reduction in venous return to the left ventricle.

(2) The reduction in blood pressure should not lead to a significant increase in the discharge from the left ventricle.

(3) Amyl nitrite itself should not produce significant changes in the elasticity of the large vessels.

Hæmodynamic studies of the effect of nitrites in the first phase have not been performed since they would require methods that could follow changes from one heart beat to the next. The reduction in venous return after nitrites (Weiss et al., 1937; Wilkins et al., 1937) occurs in the second or third phase of their effect when the moderator reflexes are acting most strongly. Animal experiments have shown (Conway, 1955) that maximal depression $\mathrm{cf}$ blood pressure can be produced by nitroglycerine with little change in pulse pressure if the regulatory reflexes are blocked by hexamethonium, indicating that nitrites do not of themselves diminish venous return and consequently cardiac output. This finding has been confirmed in further unpublished animal experiments in which the effect of nitrites has been studied with a cardiometer. With the reduction of pressure, the stroke volume was not reduced but, on the contrary, increased slightly. The possibility therefore exists that in some patients the test might be invalidated because the stroke volume increased and thus obscured a reduction in pulse pressure. It was thought, however, that in the absence of heart failure this complication would be insignificant, since changes in pulse pressure were measured from one beat to the next and the reduction in diastolic pressure between beats was small, varying from 1 to $4 \mathrm{~mm}$. $\mathrm{Hg}$. On theoretical grounds, therefore, and after some preliminary experiments it was thought that none of the factors enumerated would change sufficiently to affect the test.

The theoretical background of the test requires separate consideration since it depends upon an analysis of the physical properties of arteries. A strip of perfectly elastic material would show a linear/length relationship, as required by Hooke's law, and as shown schematically in Fig. 3 (solid line). Derivation of the pressure/volume relationship of a vessel made of this material is unexpectedly complicated, since both pressure and volume are a function of the radius of the vessel, but it can be shown mathematically that the volume will rise steeply with equal increments of pressure, as in Fig. 4 (solid line). Arteries, like other biological tissues, are never perfectly elastic (Roy, 1880) but in the arteries of young animals there is a tendency to obey Hooke's law at lower ranges of pressure, but this is lost as the pressure is raised above the normal level. This is shown graphically by the dotted lines in the illustrations, where the sigmoid character of the pressure volume curve is characteristic of the findings obtained in the young by Roy (1880), Hallock and Benson (1937), and Remington et al. (1948). The failure to obey Hooke's law is progressively greater as the pressure is raised, owing to the fact that the tension is thrown on to the collagen fibres, particularly in the adventitia, which are inelastic. From the point of view of the present test, however, it will be seen that for elastic arteries over a wide range of pressure equal increments of volume would give approximately equal changes in pressure. In the young, and in normally elastic arteries, there should be little change in pulse pressure as the diastolic pressure is reduced providing the stroke volume remained unchanged. With ageing, the level of pressure at which the artery becomes inelastic is greatly reduced, and eventually it is seen that the artery becomes inelastic even at very low pressures and the pressure/volume curve differs greatly from that obtained in the young (Fig. 5). Equal increments of volume result in greater and greater increases in pressure. In the elderly, therefore, if the stroke volume is held constant and the diastolic pressure is reduced, the pulse pressure will also be reduced (Fig. 5).

The results of the test with amyl nitrite in normal subjects showed a small reduction in pulse 


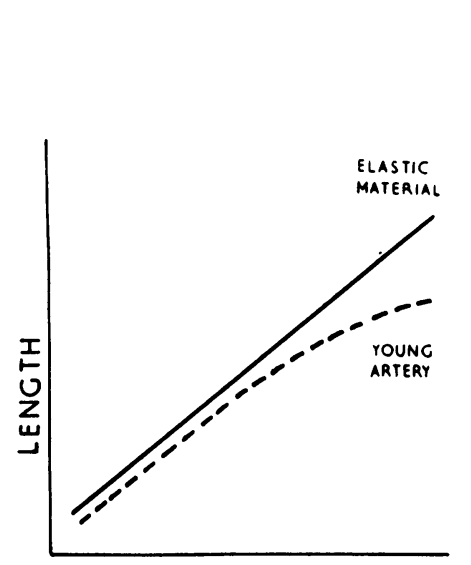

TENSION

Fig. 3.-Tension/length diagram of a strip of elastic material (solid line) and a strip of artery from a young person (dotted line).

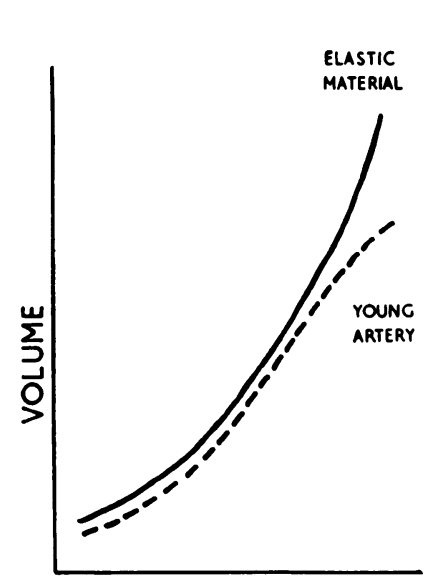

PRE SSURE

Fig. 4.-The conversion of curves in Fig. 3 for an elastic material and a young artery into a pressure/volume diagram.

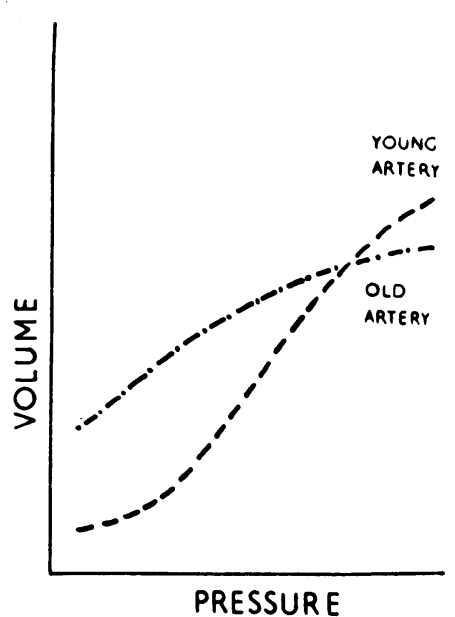

Fig. 5.-The pressure/volume relationship of arteries of young and old subjects.

pressure with the falling diastolic pressure and if the assumptions are valid, this indicates that their arteries were moderately elastic. This was also true of the "elastic" hypertensive group and the clinical findings in this group agreed with the findings in the test. The clear reduction in pulse pressure with the falling diastolic pressure in the remainder of those with hypertension indicated that their arteries were inelastic and this was confirmed in a general way by their greater age and greater initial pulse pressure, and this group included cases of arteriosclerotic hypertension. In most patients, the clinical features alone would not have been sufficient to assign them to one group or to another. No attempt has been made to assess the extent of the degenerative change in the arterial reservoir, and the linits of the elastic group have been determined by the findings in the normal subjects. More precise classification should be possible when we have applied the method more extensively. For the present, the term " inelastic" has been used in preference to "sclerotic" since it was appreciated that some cases might appear to be inelastic, not because of fibrous changes in their arteries but because the diastolic pressure might be high enough to throw considerable tension on the inelastic collagen material of the adventitia.

Although this test has been worked out on patients with hypertension among whom it has been useful in the separation of two distinct types, it is hoped that in providing a clinical method of assessing the process of ageing in arteries it may be equally useful in other arterial diseases. It may thus be possible to clarify the association between the elevation of blood pressure with age and loss of elasticity. It also offers an opportunity of studying the elastic state of the large arteries in coronary and cerebral arterial diseases, and of determining whether an association exists between the atheromatous arterial diseases and loss of elasticity.

\section{SUMMARY}

Immediately after the inhalation of amyl nitrite, the blood pressure may be seen to fall for a certain number of heart beats without change in heart rate. If it can be assumed that there is no significant change in stroke volume at this time, changes in pulse pressure may be used to indicate the elasticity of the large arteries.

The feasibility of using a test based upon this principle has been studied in 15 normal young subjects and in 37 patients with hypertension. In the young, as was predicted from theoretical 
considerations, the reduction in diastolic pressure was accompanied by little change in pulse pressure, the average being 16 per cent of the fall in diastolic pressure. In the group of patients with hypertension two types of response were seen. In one group the average reduction in pulse pressure was quite small, 30 per cent of the reduction in diastolic pressure, while in the other it almost equalled the reduction in diastolic pressure, an average of 97 per cent. The former has been called the "elastic" and the latter the "inelastic" hypertensive group. The clinical data were in general agreement with this classification in that the patients of the inelastic group were significantly older than those in the elastic group and their initial pulse pressure was also significantly greater.

We should like to acknowledge the help and co-operation of the students who volunteered for these tests, the physicians of Charing Cross Hospital who gave access to their cases, and of Miss Anne Smith, cardiographic technician, and Mr. W. Penn for their conscientious technical assistance.

\section{REFERENCES}

Bramwell, J. C. (1924). Quart. J. Med., 17, 225.

- Downing, A. C., and Hill, A. V. (1923a). Heart., 10, 289. - -, and Hill, A V. (1922). Proc. Roy. Soc., B. 93, 298.

- McDowall, R. J. S., and McSwiney, B. A. (1923c). Proc. Roy. Soc., B. 94, 450.

Conway, J. (1955). Clin. Sci., 14, 625.

Hallock, P., and Benson, I. C. (1937). J. clin. Invest., 16, 595.

Hamilton, M., Pickering, G. W., Fraser Roberts, J. A. F., and Sowry, G. S. C. (1954). Clin. Sci., 13, 11.

Master, A. M., Marks, H. H., and Dack, S. (1943). J. Amer. med. Ass., 121, 1251.

Miall, W. E., and Oldham, P. D. (1955). Clin. Sci., 14, 459.

Remington, W. J., Noback, C. R., Hamilton, W. F., and Gold, J. J. (1948). Amer. J. Physiol., $153,298$.

Roy, C. S. (1880). J. Physiol., 3,125.

Weiss, S., Wilkins, R. W., and Haynes, F. W. (1937). J. clin. Invest., 16, 73.

Wilkins, R. W., Haynes, F. W., and Weiss, S. (1937). J. clin. Invest., 16, 85. 